\title{
Experimental analysis of suction on step-induced boundary-layer transition
}

\author{
Benjamin Dimond ${ }^{\mathrm{a}, *}$, Marco Costantini ${ }^{\mathrm{a}}$, Steffen Risius $^{\mathrm{a}}$, Carsten Fuchs $^{\mathrm{a}}$, Christian Klein $^{\mathrm{a}}$ \\ ${ }^{a}$ DLR, German Aerospace Center \\ Bunsenstraße 10, 37073 Göttingen
}

\begin{abstract}
The influence of suction on step-induced boundary-layer transition has been experimentally investigated in the Cryogenic Ludwieg-Tube Goettingen at large chord Reynolds numbers (up to $16 \cdot 10^{6}$ ), Mach numbers from 0.35 to 0.77 and various streamwise pressure gradients by means of temperature-sensitive paint. Surface imperfections, implemented as combination of gap and forward-facing step, caused transition to occur at a location more upstream than in the case of a smooth surface (i.e. without gap and step). For this combination of imperfections, it was demonstrated for the first time in experiments that suction, achieved passively by exploiting the pressure difference between upper and lower side of the model, induced a movement of transition to a more downstream location than without suction, and in most cases even more downstream than on the smooth configuration at the same test conditions. Thus, the effect of suction was to even overcompensate the adverse effect of the combination of gap and forward-facing step on boundary-layer transition for the investigated test conditions.
\end{abstract}

\section{Introduction}

Laminar flow technology is of great interest as it can significantly reduce wall shear stress and therefore fuel consumption of commercial aircraft as opposed to wings of 5 conventional aircraft with predominantly turbulent flow. According to reference [1, almost $50 \%$ of all aerodynamic drag arises from friction, yielding a high possibility for fuel savings by maintaining the flow laminar over a significant portion of the wing surface. One method to delay boundary-layer transition and thus extend the area of laminar flow is an appropriate wing contour design (natural laminar flow - NLF), another method is by means of suction (laminar flow control - LFC). The former has been demonstrated to be a suitable technology for aerody15 namic surfaces with zero to moderate sweep angles (see e.g. refs. [2, 3] ) and is a practical reality for gliders and busi-

\footnotetext{
* Corresponding author

Email address: benjamin.dimond@dlr.de (Benjamin Dimond) 35
}

ness jets as described in refs. 4, 5]. At larger sweep angles, however, LFC is required to achieve large laminar flow areas according to refs. [2, 3, 6]. A promising technique lays in the combination of both methods, applying suction to a NLF-wing design. This technology, called HFLC - hybrid laminar flow control, has already been extensively investigated [6] and is starting to be implemented in commercial aircraft [7]. As reported in refs. [6, 8], flow control by means of suction has mainly been studied and tested with perforated panels providing an approximately evenly distributed suction over a large region. Early studies, like those in the 1940 s to 1960 s, also experimented with suction through slits on a flat surface (see e.g. ref. [9] or [10]). Furthermore, there have been several studies on the effect of suction on transition in the absence of steps (see e.g. the reviews in ref. [6] and [10]). Despite the promising results already achieved with laminar flow technology, its practical application remains challenging in the presence of surface imperfections at structural joints, such as gaps 
and/or steps, which are unavoidable on real aircraft [2]. The influence on boundary-layer transition due to surface imperfections has been investigated experimentally for example by refs. [11 13 and numerically by refs. [13, 14] 75 40 and [15. However, the influence of suction on transition of a two-dimensional boundary layer in the presence of steps has only been examined in two groups of studies: ref. [16] experimentally investigated the effect of suction through gaps (i.e. slits) downstream of a backward facing step at 80 low Mach numbers $(\mathrm{M}<0.1)$; numerical studies have been conducted to examine suction through a gap upstream of a forward-facing step in refs. [17, [18] and 19] but only for one Mach number $(M=0.6)$. Both studies however, only examine cases with zero pressure gradient and without variation of the Mach number (or only for a small range). In contrast, this work systematically examines the effect on transition location of suction through a gap directly upstream of a forward-facing step for a wide range of Mach numbers (0.35-0.77), Reynolds numbers (chord Reynolds numbers $3.5-16 \cdot 10^{6}$ ) and streamwise pressuregradients. Three step heights are investigated and compared with each other as well as with a smooth reference configuration without step, gap and suction. A study investigating the effect of the variation of suction rate for

${ }_{60}$ a fixed step height is presented in ref. [20]. The current article builds on results and experiments performed and discussed in ref. [21].

\section{Experimental Setup}

\subsection{Cryogenic Ludwieg-Tube Göttingen}

The experiments for the study presented in this work were conducted in the low-turbulence Cryogenic LudwiegTube Göttingen (DNW-KRG) which uses gaseous nitrogen as test gas 22, 23]. Due to the fast expansion of the ${ }_{105}$ gas during a run in the Ludwieg-Tube and the resulting than one [23, 24]. By increasing the pressure (up to 10 $\mathrm{MPa}$ ) and decreasing the temperature of the gas (possible down to $105 \mathrm{~K}$ ), large Reynolds and Mach numbers are achievable. The pressure gradient was varied by altering the angle-of-attack of the wind-tunnel model that was clamped into turntables mounted in the side walls of the test section. The test section is $0.4 \mathrm{~m}$ wide, $0.35 \mathrm{~m}$ high and $2 \mathrm{~m}$ long with upper and lower wall adapted to allow for interference-free measurements 23. The mass flux turbulence value of the facility is $T u_{\rho u} \approx 0.06 \%[22$. Further details on the wind tunnel and its instrumentation can be found in refs. 22 24.

\subsection{Wind tunnel model}

Figure 1 depicts the two-dimensional wind tunnel model PaLASTra used for the experiments. It was designed to achieve a large area of uniform pressure gradient along the model's chord on the model's upper side ([12, 24, 25]), which is the surface of interest in the present work. In the mid-span region of the model, pressure taps were installed to obtain a chordwise pressure distribution. The upper side was coated with temperature-sensitive paint (TSP) for transition detection ([26, 27]) - paint composition and optical setup was the same as in ref. [24]. The model is coated from the leading edge (included) to $x / c=0.975$ with a bare strip, where no TSP was coated, between $0.335 \leq x / c \leq 0.365$ to ensure sharp edges at the step and gap location. An additional aft part was attached to the original model (see figure 1 left) to reduce the size of separation region and thus minimize external disturbances to the boundary layer on the model's upper side as discussed in ref. 28. The chord length of the model for this study will be stated as $c=200 \mathrm{~mm}$ despite the extension with the aft part to be able to compare results to earlier studies. The joints between main part and aft part (upper and lower side) were expected to be small enough to not significantly alter the transition location in the region of interest. Shims of appropriate thickness were installed between the perature and adiabatic wall temperature $T_{w} / T_{a w}$ is larger 


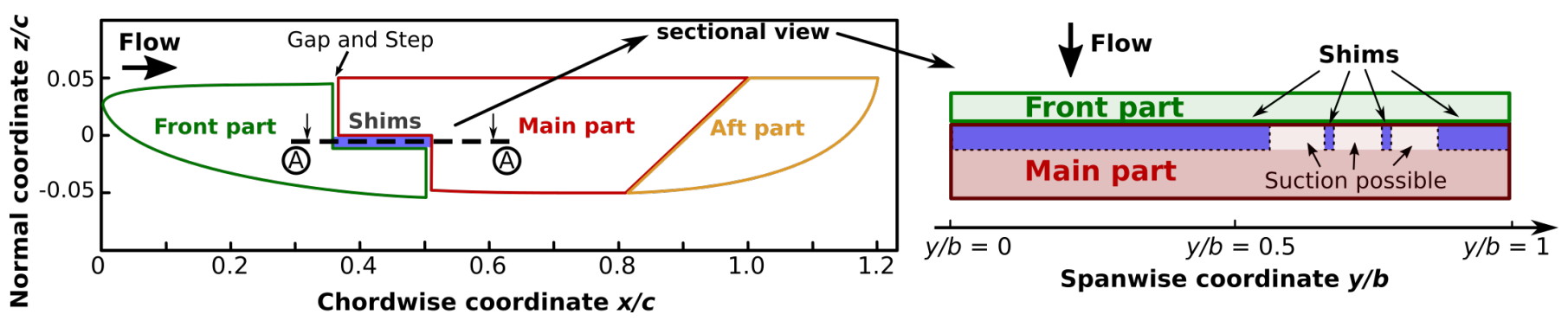

Figure 1: Simplified sketch of the PaLASTra wind tunnel model (chord length $c=200 \mathrm{~mm}$, span $b=500 \mathrm{~mm}$ ). The left sketch is a side view of the whole model cross-section, whereas the right sketch is a sectional view in the A-A plane looking from the top. Shim sizes are in proportion, dimensions of gap and step are enlarged for better visibility.

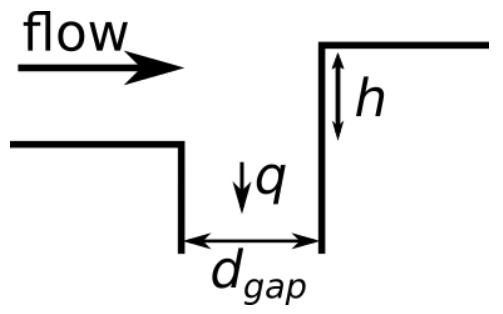

Figure 2: Illustration of step (height: $h$ ) and gap (width: $d_{\text {gap }}$ ) at the junction between front and main part of the model.

front and main part of the model to obtain sharp forward-

facing steps at $35 \%$ of the model's chord length ([12]).

Along with a nominally smooth configuration (i.e. with

a shim thickness resulting in a smooth surface without $\mathrm{a}_{140}$ step), three step heights ( $h=0 \mu \mathrm{m}, h=30 \mu \mathrm{m}$ and $60 \mu \mathrm{m}$ - see figure 2 were investigated. For these configurations, the main part of the model was additionally displaced in the streamwise direction using alignment pins to obtain a gap width of $d_{\text {gap }}=200 \mu \mathrm{m}$ upstream of the steps. The $\mathrm{T}_{145}$ step and gap dimensions were determined with a contact profilometer (vertical resolution $\pm 8 \mathrm{~nm}$ ). The detected spanwise variation in step height $h$ was below $2 \mu \mathrm{m}$ and in gap width $d_{\text {gap }}$ below $10 \mu \mathrm{m}$.

In this work, the model configurations will be named ${ }_{150}$ "smooth" (no step and gap), "step-0" (0 $\mu \mathrm{m})$, "step$1 "(30 \mu \mathrm{m})$ and "step-2" $(60 \mu \mathrm{m})$. The correspond25 ing step Reynolds numbers were, for example, $R e_{h}=$ $U_{\infty} h / \nu_{\infty} \sim 1200$ (step-1) and $R e_{h} \sim 2400$ (step-2) for a chord Reynolds number of $R e_{c}=8 \cdot 10^{6}$. Here, $U_{\infty}$ is the freestream velocity and $\nu_{\infty}$ is the freestream kinematic viscosity. It must however be noted here that due to model 130 preparation between the measurements of the step- 1 and step-2 configuration both sides of the gap were slightly rounded and can no longer be described as sharp edges. It cannot be fully excluded that this rounding has an influence on transition location. Reproduction measurements however showed no variation within the error range of detected transition location between cases with rounded and nominally sharp edge.

As sketched in figure 1, right, narrow shims were used as placeholders on the starboard side of the model, whereas a continuous shim was used on the port side. For the investigated test cases, the examined model cross section induces a larger pressure on the model's upper side than on the model's lower side. This pressure difference induces an internal flow (suction for the model's upper side) through the gap (slit) in the regions of the model starboard side where no shims are present. For comparison, a continuous shim on the port half of the model did not allow passive suction. In this way, the effect of the combination of a gap and a forward-facing step on boundary-layer transition could be examined simultaneously with and without suction through the gap. 


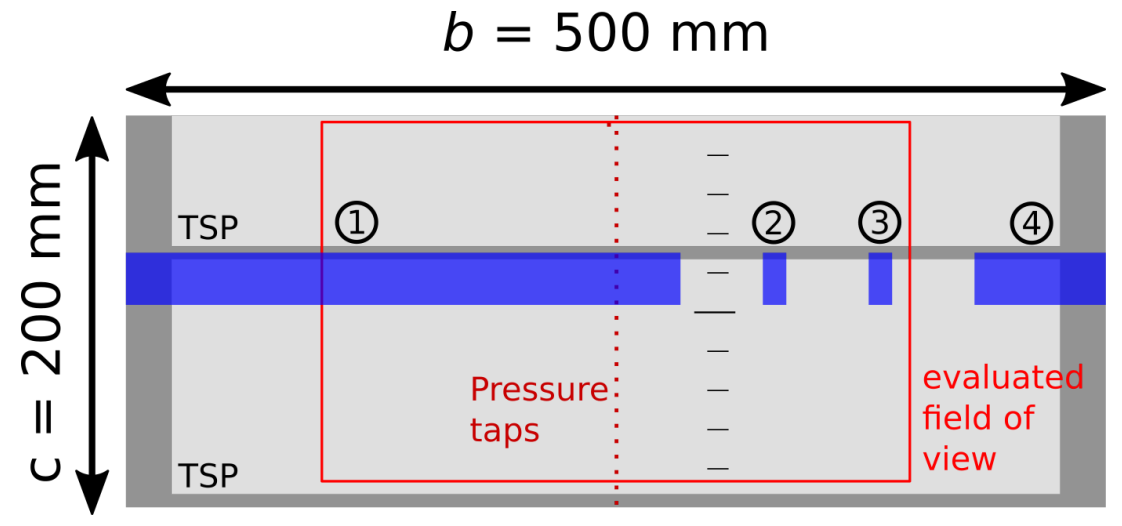

(a)

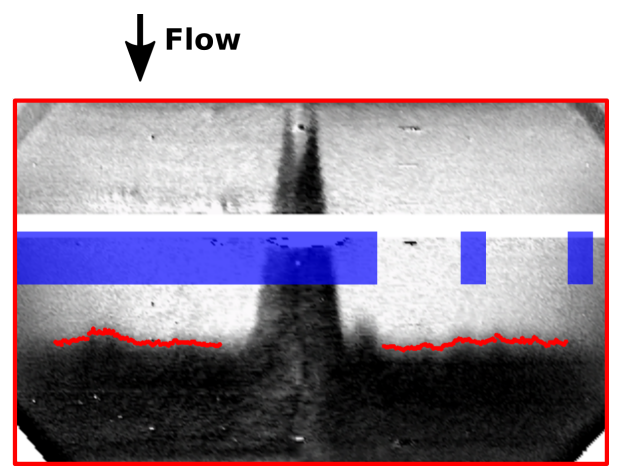

(b)

Figure 3: Position and dimension of the shims (blue) in (a) top view sketch of the whole model and (b) of the TSP results obtained from the evaluated area (marked as red rectangle). Shim dimensions and positions are in proportion. Flow conditions for (b) were $M=0.65, R e_{c}=8 \cdot 10^{6}$ and $\mathrm{AoA}=-1.9^{\circ}\left(\beta_{H}=0.064 \pm 0.003\right)$.

\section{Data Analysis}

\subsection{Transition detection}

Figure 3 depicts a top view of the model (a) and a typof the different convective heat flows of laminar and turbulent boundary layers and because the free stream has a lower temperature compared to the model temperature due to expansion of the gas in DNW-KRG, a different turbulent flow: lower convection of the laminar boundary layer causes laminar regions to cool down significantly slower compared to regions with turbulent flow regime. This can be exploited with temperature-sensitive paint to ers [27]. In the TSP images, bright areas correspond to laminar regions, whereas dark areas correspond to turbulent regions. In blue the positions and dimensions of the shims are displayed in fig. 3. On the starboard side of the ical TSP result (b) for the smooth configuration. Because ${ }_{180}$ temperature is imposed on the model for laminar and for $_{185}$ obtain transition from laminar to turbulent boundary lay-190 model, tick markers (every $10 \%$ of the chord length) can be ${ }_{195}$ seen. Because of the confined environment of DNW-KRG and the resulting limited camera angle (compare ref. [12]), only the area within the red rectangle is evaluated. Images from two cameras installed in the side walls of the test section are merged and transformed to obtain a virtual birds eye view (compare fig. 3b). To increase the reliability and productivity, an automatic data acquisition system for the intensity-based TSP method has been developed, based on the DLR software package ToPas [29]. Evaluation was therefore possible in an automated way on a virtual 3D grid of the model and does not rely on manual extrapolation based on the tick markers. Two turbulent wedges in the central area of the model can be observed, originating from pressure taps installed on the leading edge region. No TSP had been applied over the white strip visible in figure $3 \mathrm{~b}$ the junction between front and main part of the model is located in this region, which was left uncoated to enable the generation of sharp steps (compare figure $3 \mathrm{a}$ ) as stated in refs. [12, 30].

Transition detection was carried out using the streamwise maximum temperature gradient technique described in ref. [12]. Similar to earlier studies $([25])$, transition was detected at up to 100 positions on each side (port and starboard) of the model, marked by individual red dots in figure $3 \mathrm{~b}$ (appearing as red line). Excluded from the evaluation are areas of turbulent wedges or areas on the outer edges of the model which are effected by side wall effects. For both sides of the model an average transition location with according RMS of the variation was calculated. To 
easily, but also more robustly and reliably compare transi-235 tion locations for different conditions and configurations, the mean values of transition location (with corresponding RMS as uncertainty) will be used as single value in the following even though there is a spatial dispersion of the transition process itself.

\subsection{Determination of suction rate}

Because the suction is induced passively, the suction rate cannot be fixed to a set value. Instead, it depends on various parameters including gap width, free stream
Mach number, angle-of-attack and charge pressure. Pre- ${ }_{245}$ vious studies like those presented in refs. [20, 31] relied on the pressure difference between model's upper and lower side to estimate the suction rate. In those estimations it was not possible to accurately account for channel inlet and outlet or the precise influence of all of the channel ${ }_{250}$ geometry. So for this study additional pressure taps were installed along the gap inside of the model. They are located between the second and third shim as numbered in figure 3 This allowed to obtain a more accurate estimation of suction velocity with the pressure loss along a straight ${ }_{255}$ channel without needing to take into account the geometrical aspects mentioned above. The average suction velocity inside the gap can be estimated with 32

$$
v=\sqrt{\frac{2 \Delta p d_{H}}{\rho \lambda l}},
$$

25 where $\lambda=0.3164 / \sqrt[4]{R e_{d_{H}}}$ is the friction coefficient, $d_{H}=$ $2 \cdot d_{\text {gap }}$ is the hydraulic diameter, $\rho$ the density and $l$ and $\Delta p$ distance and pressure difference between two pressure taps respectively. This is valid for incompressible, turbulent channel flow with smooth walls as is reasonably ${ }^{265}$ assumed in this case (channel Reynolds numbers are calculated to be larger than 3000). The average suction velocity can then be used to express a dimensionless suction parameter defined as

$$
q=\frac{v \cdot d_{g a p}}{\delta^{*} \cdot U_{\infty}}
$$

with freestream velocity $U_{\infty}$ and displacement thickness $\delta^{*}$, determined with the boundary-layer solver COCO (see section 3.3 calculated for the smooth configuration at the junction location. For the investigated test cases presented here, the determined uncertainty of the suction parameter $q$ is less than $<10 \%$.

\subsection{Linear stability analysis}

Using the compressible boundary-layer solver COCO [33, which uses the determined surface temperature and pressure distribution, laminar boundary-layer computations were performed. The calculated boundary-layer data are used to perform a local linear stability analysis with LILO 34, calculating the amplification ratios of TollmienSchlichting waves (TS-waves). For the 2D-model used in this investigation and examined at $M=0.35$ to 0.77 , TSwaves are expected to be mainly responsible for boundarylayer transition, as discussed in [12. Amplification ratios are expressed in $\mathrm{N}$-factors where $e^{N}=A / A_{0}$ with amplitude $A$ and initial amplitude $A_{0}$ of the TS-waves. The initial amplitude $A_{0}$ is determined by receptivity processes which are not considered in this method. An envelope of the most amplified TS-waves for each location is used to obtain the critical N-factor and frequency: The most amplified TS-wave at the transition location is utilized for their determination.

Even though COCO is capable of implying suction as stability modifier (designed for low distributed suction rates over a large part of the model's chord), it is based on the boundary-layer assumptions, which may not be valid in the case of high local suction rates considered here. Similarly, the assumptions of linear, local stability theory may not apply to the investigated cases with high local suction rates. Moreover, in the presence of the steps, the step-induced variations in the flowfield and the related variations in the disturbance amplification ratios cannot be captured with the used numerical tools. Nevertheless, at the suction location, it is possible that the Tollmien- 
be interpreted cautiously for the reasons presented above. Note that the boundary-layer calculations mostly could

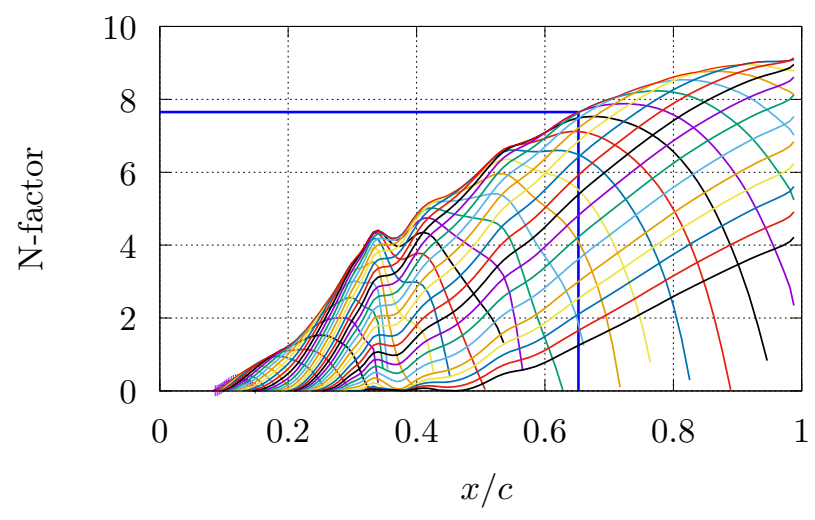

(a)

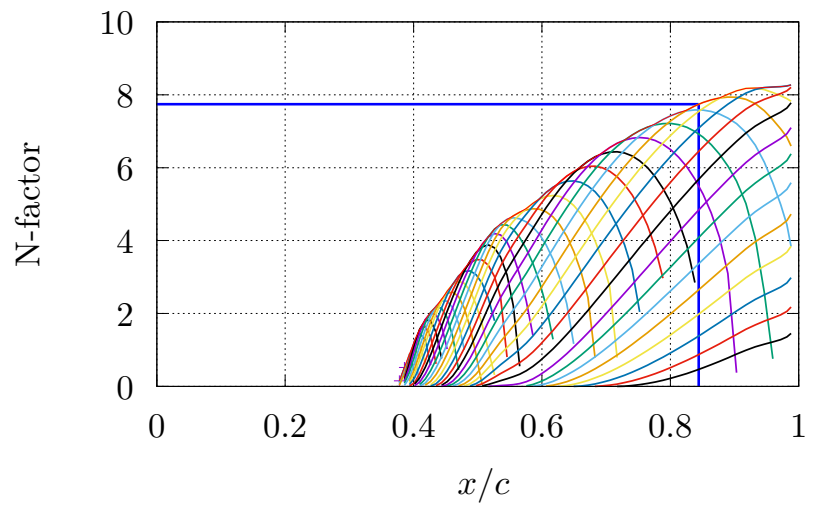

(b)
Figure 4: Comparison of calculated amplification ratios of various Tollmien-Schlichting frequencies for the smooth configuration without suction (a) and with step-2 configuration and suction (b). Blue line indicates transition location and critical N-factor. For figure (b), boundary-layer computations and linear stability calculations were started at the step and gap location $(x / c=0.35)$, therefore the computed N-factors should be considered as estimations. The corresponding TSP images are presented in figure 6 with $M=0.65, R e_{c}=8 \cdot 10^{6}$ and $\beta_{H}=0.065 \pm 0.004$. (a): Transition experimentally detected at $x_{T} / c=65 \%$ with most amplified TSfrequency $21780 \mathrm{~Hz}$ and critical N-factor 7.7. (b): Transition experimentally detected at $x_{T} / c=84 \%$ with most amplified TS-frequency $26475 \mathrm{~Hz}$ and critical N-factor 7.7.

Schlichting waves are fully damped and then re-amplified after the step as well as that completely new disturbancess10 are coupled into the boundary-layer. Therefore, results obtained via linear stability computations performed starting from the step and gap location may still be meaningful estimations of the amplification ratios, but they must not be performed accurately because of the high suction rates leading to singularities in the calculations. Two series of calculations were therefore performed: one with including suction in the boundary-layer solver COCO but starting the stability calculations only from the step and gap location (option I) and another one also starting the boundary-layer calculations only at the step and gap location (option II). Option II assumes the boundary layer to start only at the step and gap location but with the measured pressure and temperature distribution. Both calculations delivered very similar results (with critical $\Delta N$ at the experimentally measured transition location typically well below 0.5). Therefore option II was used to avoid convergence problems in the boundary-layer computations due to the high suction rates. Figure 4 illustrates two example calculations with the smooth (a) and step2 (b) configuration. As discussed above, calculations for the step-2 configurations were performed beginning at the step and gap location and the obtained amplification factors of TS-waves should be regarded as estimations. For both cases (smooth and step-2 configurations) the critical N-factor was identified as 7.7 for the experimentally detected transition locations.

\subsection{Pressure gradient parameter}

Figure 5 shows a typical chordwise pressure distribution of the wind-tunnel model PaLASTra with additional aft part for the smooth configuration (i.e. without step and gap) determined with the pressure taps described in section 2.2. The variations occurring around $x / c=0.35$ are due to the junction of the model in this area that couldn't be completely avoided (these variations also effect the stability calculation as can be seen in figure 4 (a)). To describe the pressure distribution in one quantity, the dimensionless Hartree parameter $\beta_{H}$ was chosen. It originates from boundary-layer theory for incompressible wedge flows 


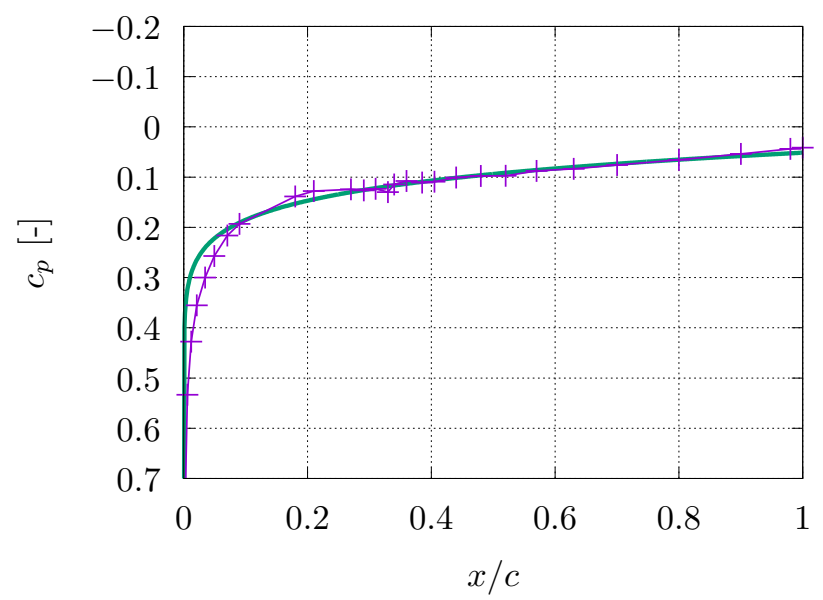

Figure 5: Chordwise pressure distribution for the smooth configuration on the upper side of the model and fitted wedge flow pressure distribution between $x / c=0.05$ and $x / c=0.9$ with Hartree paramter $\beta_{H}$, where $M=0.65, R e_{c}=8 \cdot 10^{6}$ and angle-of-attack $\alpha=1.90 . \beta_{H}=0.064 \pm 0.003$ was determined.

with wedge angle $\pi \beta_{H}$. It can be determined by 35 .

$$
\beta_{H}=\left(\frac{1}{2}-\frac{1-c_{p}(x / c)}{(x / c) \frac{\partial c_{p}}{\partial(x / c)}}\right)^{-1}
$$

with pressure coefficient $c_{p}$ and is fitted to the pressure distribution between $x / c=0.05$ and $x / c=0.9$ (see figure 5p. In this region previous studies proved a self- similar boundary-layer velocity profile 25. The wedge ${ }_{340}$ flow pressure distribution, with $\beta_{H}$ fitted to the measured pressure distribution seen in figure 5 is shown in green. It shows a high accordance in the main part of the model where the model is flat, with significant deviations only in the leading-edge region. The uncertainties of the Hartree parameter are therefore not just based on 345 inaccuracies of the pressure measurement itself but also on deviations from wedge flows due to the rounded leading edge of the wind-tunnel model and the Hartree parameter derived for incompressible flows. A relationship between the Hartree parameter $\beta_{H}$ and the incompress-350 ible shape factor $H_{12, \text { inc. }}=\delta^{*} / \delta_{2}$, ratio of displacement thickness $\delta^{*}$ and momentum thickness $\delta_{2}$, can be expressed as $H_{12, \text { inc. }} \approx-0.912 \cdot \beta_{H}+2.610$, where $H_{12, \text { inc }}$ was determined by averaging the shape factor calculated with

\begin{tabular}{|c|c|c|c|c|}
\hline$R e_{c}\left[\cdot 10^{6}\right]$ & $M$ & $\begin{array}{c}R e_{h} \\
\text { step-1 }\end{array}$ & $\begin{array}{c}R e_{h} \\
\text { step-2 }\end{array}$ & $R e_{\text {gap }}$ \\
\hline 3.5 & 0.35 & 525 & 1050 & 3500 \\
\hline 4 & 0.6 & 600 & 1200 & 4000 \\
\hline 4.5 & 0.5 & 675 & 1350 & 4500 \\
\hline 6 & $\begin{array}{c}0.35,0.5,0.6, \\
0.65,0.77\end{array}$ & 900 & 1800 & 6000 \\
\hline 8 & $\begin{array}{c}0.35,0.5, \\
0.6,0.65\end{array}$ & 1200 & 2400 & 8000 \\
\hline 10 & $0.35,0.5$, & 1500 & 3000 & 10000 \\
\hline 12 & $0.6,0.65$ & & & \\
\hline 14 & 0.65 & 1800 & 3600 & 12000 \\
\hline 16 & 0.65 & 2400 & 4800 & 16000 \\
\hline
\end{tabular}

Table 1: Test conditions for the current study with corresponding step Reynolds numbers for step-1 and step-2 configuration.

the boundary-layer solver COCO between $x / c=0.24$ and $x / c=0.9$, as suggested in ref. [25].

\subsection{Test conditions}

Table 1 contains the underlying test conditions for the results presented in this study. The test conditions for a chord Reynolds number $R e_{c}=8 \cdot 10^{6}$ are analyzed and presented in more detail in the following sections.

\subsection{Reproducibility and repeatability of the results}

In this experimental investigation close attention was payed to accuracy and reproducibility of the results. This included adaptation of upper and lower walls of the test section of DNW-KRG and reproduction of the pressure distribution by adjusting the angle-of-attack after every configuration change. The accuracy levels accepted for this study were $\pm 0.1 \cdot 10^{6}$ for the chord Reynolds number and \pm 0.002 for the Mach number. The given values of these parameters in this study are all within these error ranges. The transition location could mostly be reproduced well below $x / c=0.02$ in reproducibility measurements, some of 

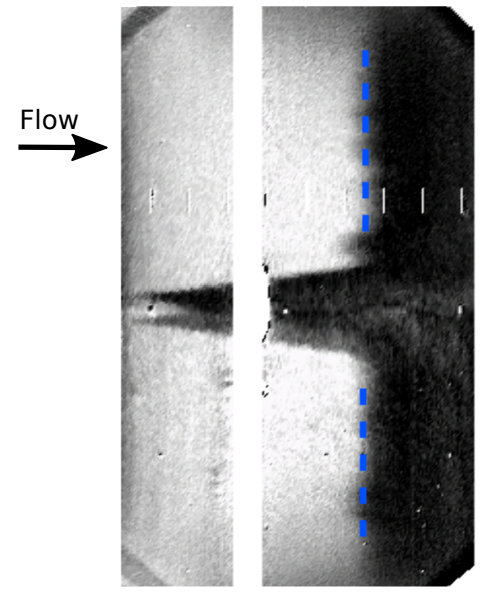

(a) $\operatorname{smooth}\left(R e_{h} \sim 0\right)$
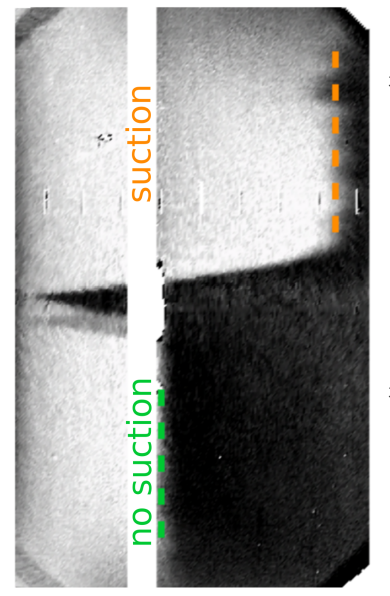

(b) step-2 $\left(R e_{h} \sim 2400\right)$
Figure 6: TSP results for nominally smooth configuration (a) and step + gap configurations with $R e_{h} \sim 2400$ and $R e_{\text {gap }}=$ $U_{\infty} d_{\text {gap }} / v_{\infty} \sim 8000$ for (b) at $M=0.65, R e_{c}=8 \cdot 10^{6}$ and $\beta_{H}=0.065 \pm 0.004$. Transition was detected at $x_{T} / c \sim 65 \%$ (smooth) for (a) and at $x_{T} / c \sim 40 \%$ (no suction) and $x_{T} / c \sim 84 \%$ (suction) for (b).

\section{Results and discussion}

Figure 6 depicts example TSP results obtained with the smooth (a) and step-2 (b) configurations at $M=0.65$, $R e_{c}=8 \cdot 10^{6}$ and a favorable pressure gradient $\left(\beta_{H}=\right.$ wise averaged transition location - blue for the smooth configuration, green for areas without suction and orange for areas with suction.

Without suction (port side), the step and gap combination upstream than in the case of the smooth configuration due to findings in refs. [12 15, 30] for steps without gaps or in refs. [36, 37] for gaps without steps as well as numerical

predictions for steps in combination with gaps as stated in 375 refs. 18, 19. For cases with suction (starboard), however, transition was shifted towards a more downstream location. It should be emphasized here, that for the examined suction rates the suction effect overcompensates the adverse effect of combination of gap and forward-facing step, since transition was measured at a more downstream location than that detected on the smooth configuration. This was predicted also in numerical studies as presented in [17]. In addition to that, note that the transition front is not as straight as without suction and appears frayed (compare figure 7). This could be due to 3D roughness at the step and gap location or due to a spanwise variation of the suction rate itself. These findings hold true for all conditions examined in the present work.

\subsection{Influence of pressure gradient parameter on transition}

Figure 7 illustrates the effect of varying the pressure gradient for $M=0.6$ and $R e_{c}=8 \cdot 10^{6}$ for the step- 1 configuration $(30 \mu \mathrm{m})$. Increasing the Hartree parameter $\beta_{H}$ (resulting in an acceleration of the boundary layer and thus stabilizing it) causes transition to occur further $0.065 \pm 0.004)$. Dashed lines indicate the detected, $\operatorname{span}^{-}{ }^{400}$ clearly induces a shift of the transition to a location more 405 to higher amplification of TS-waves. This is in agreement [36, 37] for gaps without steps as well as numerical downstream. This is true for cases with and without suction and for all examined conditions, though more prominent for cases with suction. For a configuration with step but without preceding gap the same effect was already observed in ref. 12 .

\subsection{Influence of Mach and Reynolds number on transition}

Figure 8 illustrates the effect of Mach number $(M=$ $0.35, M=0.5, M=0.65)$ on transition Reynolds number $R e_{x T}=x_{T} U_{\infty} / \nu_{\infty}$ (formed with the detected transition location $x_{T}$ ) for a chord Reynolds number $R e_{c}=8 \cdot 10^{6}$. Linear stability analysis predicts a stabilizing effect of compressibility on boundary-layer transition [38. In this investigation the opposite effect was observed for both cases with and without suction as increased Mach numbers lead to earlier transition. This was examined in ref. 25] and 


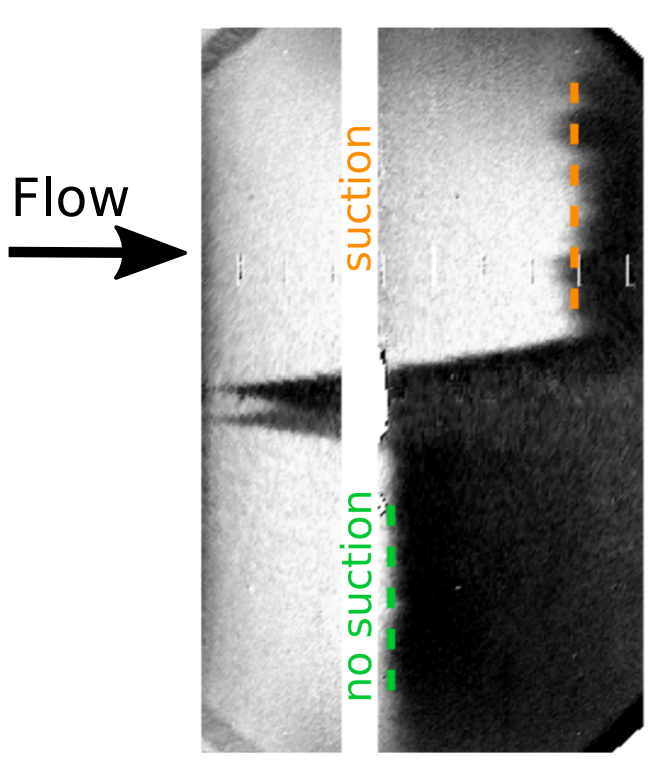

(a) $\beta_{H}=0.043 \pm 0.003$

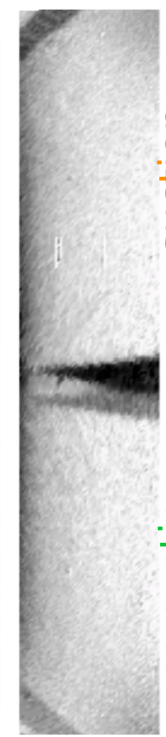

(b) $\beta_{H}=0.068 \pm 0.003$
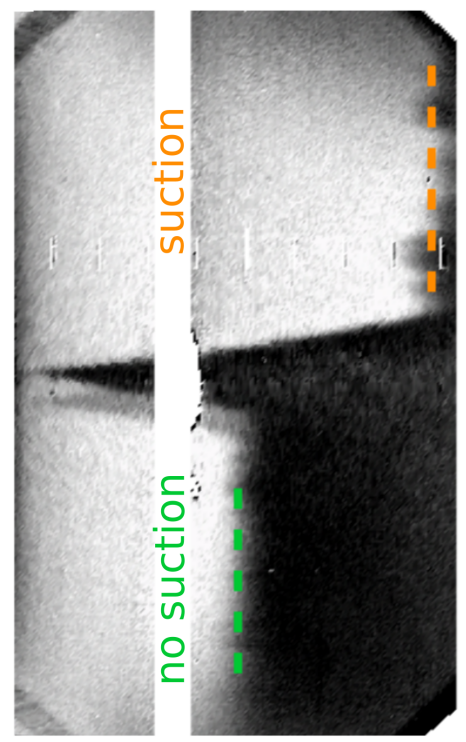

(c) $\beta_{H}=0.084 \pm 0.004$

Figure 7: TSP results for step-1 $(30 \mu \mathrm{m})$ configuration at different pressure gradients with chord Reynolds number $R e_{c}=8 \cdot 10^{6}$ and Mach number $M=0.6$ ( $R e_{g a p}=U_{\infty} d_{g a p} / v_{\infty} \sim 8000$ for all cases). Transition was detected at $x_{T} / c \sim 41 \%$ (green) and $x_{T} / c \sim 79 \%$ (orange) for (a), at $x_{T} / c \sim 46 \%$ (green) and $x_{T} / c \sim 85 \%$ (orange) for (b) and at $x_{T} / c \sim 48 \%$ (green) and $x_{T} / c \sim 88 \%$ (orange) for (c).

explained with the increased freestream disturbance level ${ }_{430}$ of the wind tunnel based on measurements of ref. [22] expected to lead to higher initial amplitudes of TS-waves. Transition Reynolds number dependence on the Hartree parameter is similar for all Mach numbers but more pronounced for cases with suction. This is similarly observed in figure 9, comparing transition Reynolds numbers for ${ }^{435}$ varying chord Reynolds numbers $\left(R e_{c}=6 \cdot 10^{6}, R e_{c}=\right.$ $\left.8 \cdot 10^{6}, R e_{c}=10 \cdot 10^{6}\right)$ at $M=0.65$. An increase in transition Reynolds number for increased chord Reynolds number was observed and explained in ref. 25] for a smooth configuration; it could also be observed for this case with ${ }^{440}$ a step and gap configuration. Transition for higher chord Reynolds numbers is caused by Tollmien-Schlichting waves with higher frequencies than for lower chord Reynolds numbers. Since freestream disturbances are lower for high frequencies in DNW-KRG 22], this leads to higher ampli-445 fications required to trigger transition for higher Reynolds numbers, thus delaying transition. This effect was observed to be more significant in the reference configuration (see figure 12). In figure 8 and figure 9 the yellow symbols represent the same data for $M=0.65$ and $R e_{c}=8 \cdot 10^{6}$. All data sets presented in these figures suggest a linear dependency within the examined Hartree-parameter range and thus a linear regression was plotted to guide the eye.

All results obtained with the smooth and step-2 configurations are collected in figure $10 \mathrm{a}$ and $10 \mathrm{~b}$, respectively. Again the transition Reynolds number is plotted against the Hartree parameter $\beta_{H}$. It can clearly be seen that for all presented test and suction conditions investigated in this study, larger $R e_{x T}$ were obtained by means of suction upstream of the forward-facing step, as compared to the smooth configuration. For the examined range of pressure gradients, however, the variation in $R e_{x T}$ for a certain variation in $\beta_{H}$ is larger with the smooth configuration than with the step- 2 configuration with suction, suggesting that the dependency on the pressure gradient is not as high as for the smooth configuration. For the largest Hartree parameters examined here $\left(\beta_{H}>0.12\right)$ this leads to the smooth configuration yielding more laminar- 


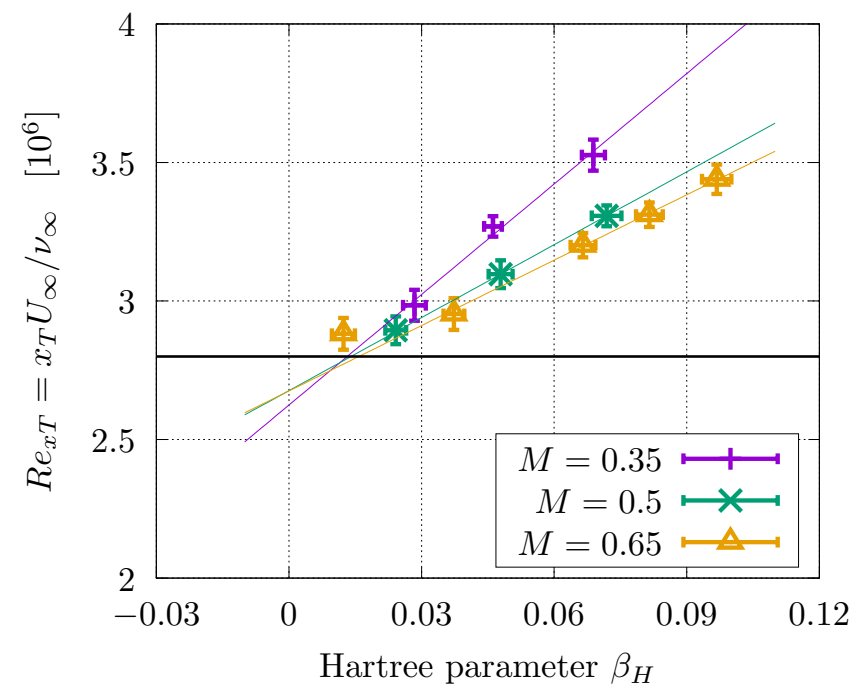

(a) step-2 - no suction

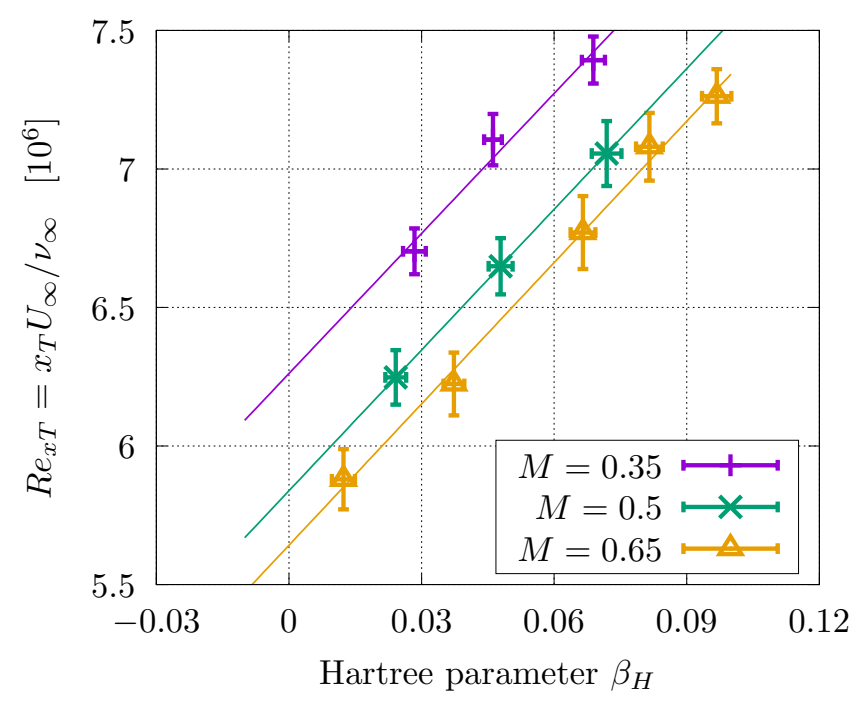

(b) step-2 - suction

Figure 8: Transition Reynolds number $R e_{x T}$ as function of the Hartree parameter $\beta_{H}$ for varying Mach numbers at $R e_{c}=8 \cdot 10^{6}$. (a) is without suction (port) and (b) with suction (starboard). The black line in (a) indicates the Reynolds number corresponding to the step and gap location. The linear regression curves were fitted without the values corresponding to the lowest Hartree parameters for $M=0.5$ and $M=0.65$ since transition was detected too close to the step and gap location. Note the small range of the y-axis, showing the Mach number effect to be relatively small.

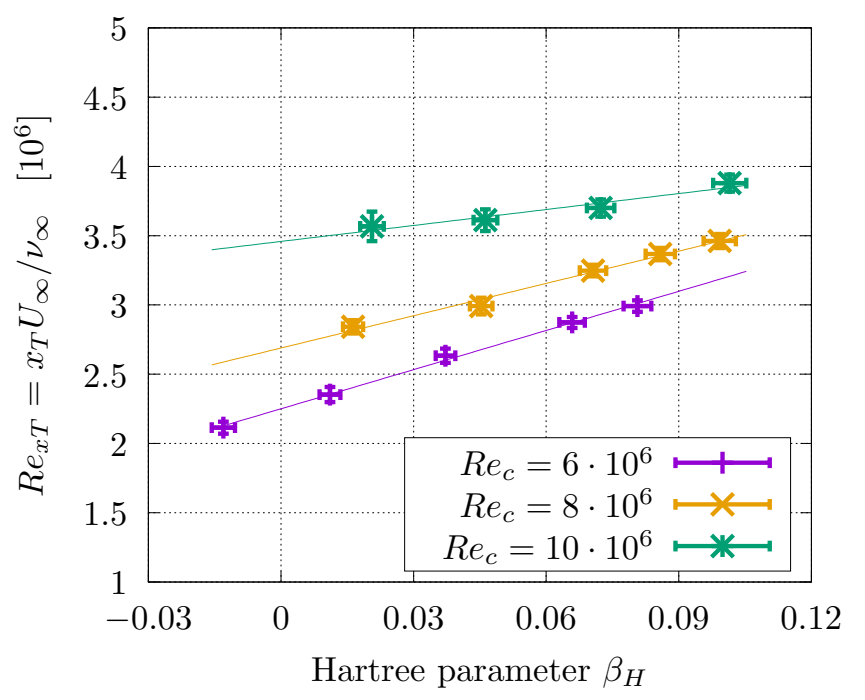

(a) step-2 - no suction

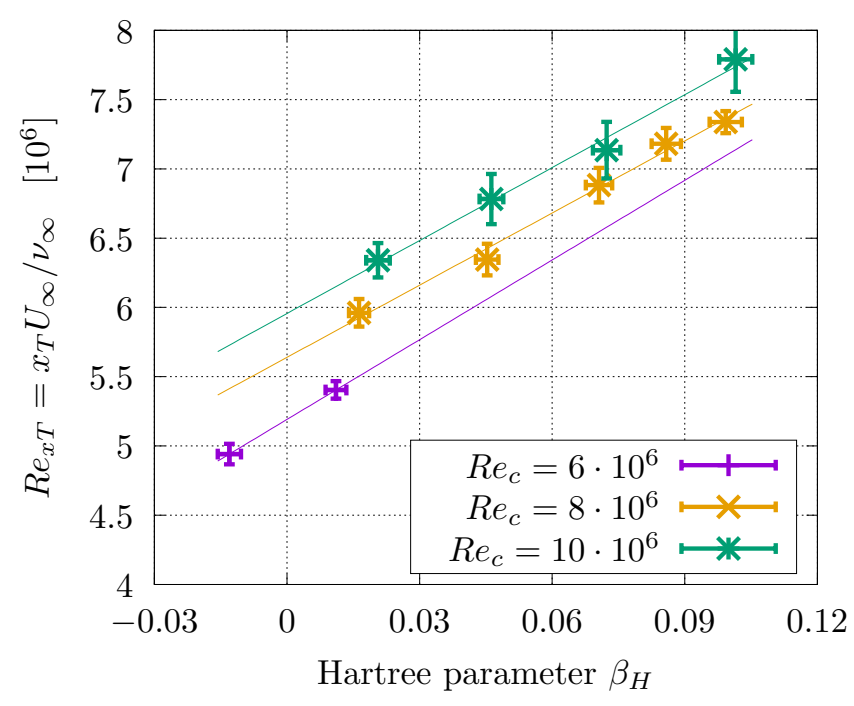

(b) step-2 - suction

Figure 9: Transition Reynolds number $R e_{x T}$ as function of the Hartree parameter $\beta_{H}$ for varying Reynolds numbers at $M=0.65$. (a) is without suction (port) and (b) with suction (starboard). 
This however, was only observed for the highest Mach and Reynolds numbers considered in this work and could not be investigated for larger $\beta_{H}$ because of the constraints for the present experimental setup discussed in ref. [12.

\subsection{Effect of step height on transition location}

In figure 11. TSP results for the step-0, step-1 and step-2 configurations are shown for $M=0.65, R e_{c}=8 \cdot 10^{6}$ and a favorable pressure gradient (in the range of $\beta_{H}=$ $0.065 \pm 0.004)$. For the port side of the model without suction, an increase in step height clearly shifts the transition location to a more upstream location. On the suction side however, this effect is not as prominent and between the step-1 and step-2 configuration almost no shift in transition location can be detected. Note, that for the step-1 and step-2 configuration the transition location on the port side of the model appears straight, whereas for the step0 configuration transition is pitched downstream towards the center of the model. This effect was similarly observed also for other flow conditions. One possible explanation is that there is a spanwise flow toward the starboard side in the upper part of the gap leading to boundary-layer suction also in parts of the port side. This could either not be the case for the step- 1 and step- 2 configuration due to different gap-channel inflow conditions and resulting suction rate (see below for details) or the effect not being sufficient to alter boundary-layer transition: a higher step height could require higher minimum suction rates than the step-0 configuration to delay transition. A low suction rate that is expected here could then only cause delay in transition location for a small step height.

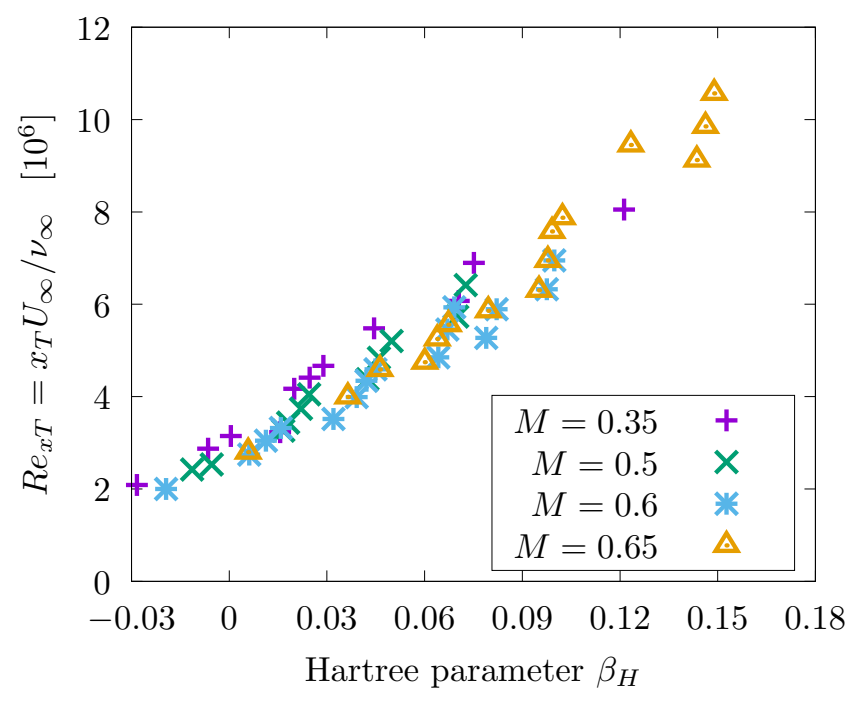

(a) smooth configuration

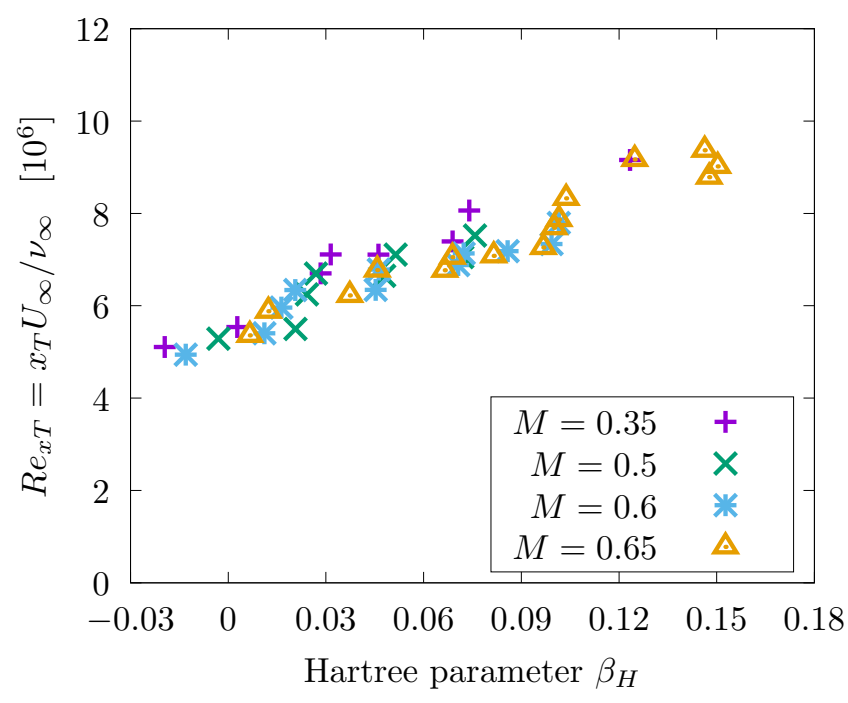

(b) step-2 configuration

Figure 10: Transition Reynolds number $R e_{x T}$ as function of the Hartree parameter $\beta_{H}$. Error bars are typically smaller than the symbols and therefore not shown here but are similar to those in figures 8 and 9 for all data points. 


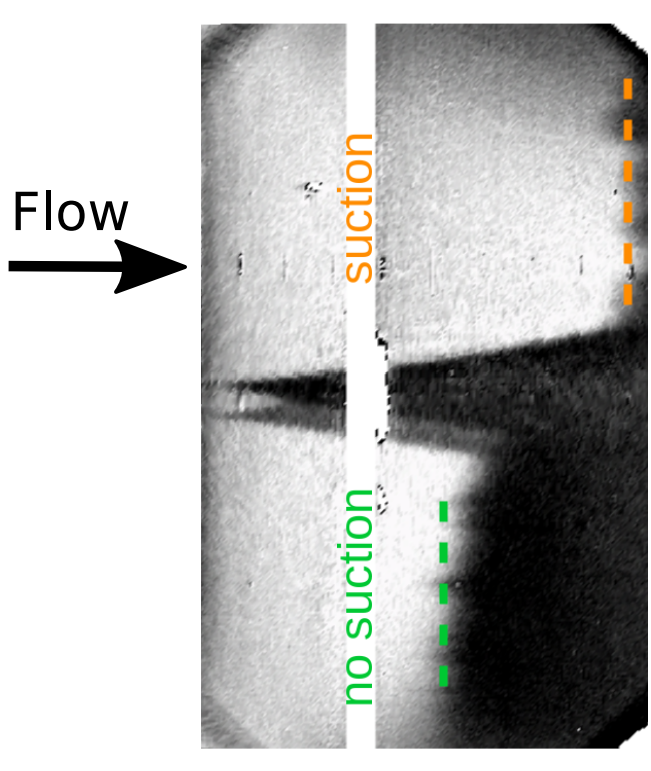

(a) $\operatorname{step}-0\left(R e_{h} \sim 0\right)$
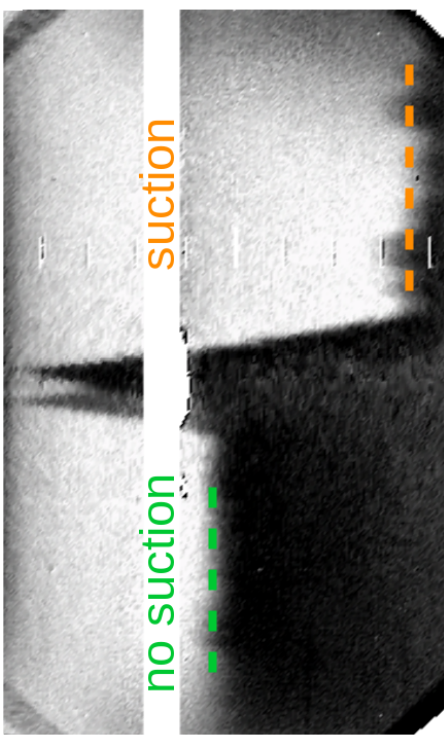

(b) step-1 $\left(R e_{h} \sim 1200\right)$
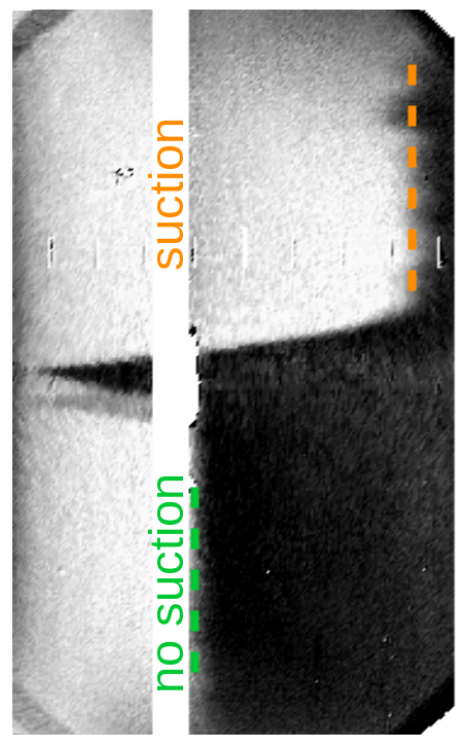

(c) $\operatorname{step}-2\left(R e_{h} \sim 2400\right)$

Figure 11: TSP results for step and gap configurations with $R e_{h} \sim 0$ (a), $R e_{h} \sim 1200$ (b) and $R e_{h} \sim 2400$ (c) $\left(\operatorname{Re}_{\text {gap }}=U_{\infty} d_{g a p} / v_{\infty} \sim 8000\right.$ for all cases) at $M=0.65, R e_{c}=8 \cdot 10^{6}$ and $\beta_{H}=0.065 \pm 0.004$. Transition was detected at $x_{T} / c \sim 52 \%$ (green) and $x_{T} / c \sim 90 \%$ (orange) for (a), at $x_{T} / c \sim 45 \%$ (green) and $x_{T} / c \sim 86 \%$ (orange) for (b) and at $x_{T} / c \sim 40 \%$ (green) and $x_{T} / c \sim 84 \%$ (orange) for (c).

The different step heights result in altering suction rates as determined with the pressure taps inside of the gap. For the step- 0 configuration it is roughly $\Delta q \approx 0.1$ lower step-2: $q \approx 0.85)$. This is probably due to the different gap-channel inflow conditions: a higher step height facilitates flow stagnation and thus increased pressure at the upper part of the gap. This yields higher suction velocities and suction rates. The step-1 configuration was not 515 investigated in this test campaign, but the suction rate is expected to be in between those of step- 0 and step- 2 configurations.

Figure 12 shows the transition Reynolds number $R e_{x T}$ versus the Hartree parameter $\beta_{H}$ with a fixed Mach num- ${ }^{520}$ ber $(M=0.6)$ and chord Reynolds number $\left(8 \cdot 10^{6}\right)$ for the different step heights with and without suction. Underlying TSP results for the step-1 configuration and three different pressure gradients are depicted in figure 7. Whereas without suction there is a significant difference of $R e_{x T}$ for ${ }^{525}$ the different step configurations at same Hartree parame- ter $\beta_{H}$ (compare also ref. [12 for steps without gap), the cases with suction yield similar $R e_{x T}$ for all three step heights, though slightly larger for the step-0 configuration. For the step-1 and step-2 configurations the detected transition locations are even essentially the same. This suggests the step height in the examined range has little influence on transition location for these suction rates and flow conditions. This finding also holds true for all other examined Mach and Reynolds numbers. An explanation could be found in the relatively high suction rate: here it is determined between $q=0.99\left(\right.$ low $\beta_{H}$ ) and $q=1.27$ (high $\left.\beta_{H}\right)$ for the step-2 configuration and between $q=0.82$ (low $\beta_{H}$ ) and $q=1.13$ (high $\beta_{H}$ ) for the step-0 configuration. This is significantly larger than the suction range examined by the numerical study in ref. [17] (with $q=0.1$ and 0.3 ) or for an experimentally investigated backward facing step (with $q<0.2$ ) in ref. [16]. The effect of the suction rate $q$ in the range $0.02 \lesssim q \lesssim 1$ on step-induced transition for the step-2 configuration is experimentally investigated in ref. [20], where it was shown that the 


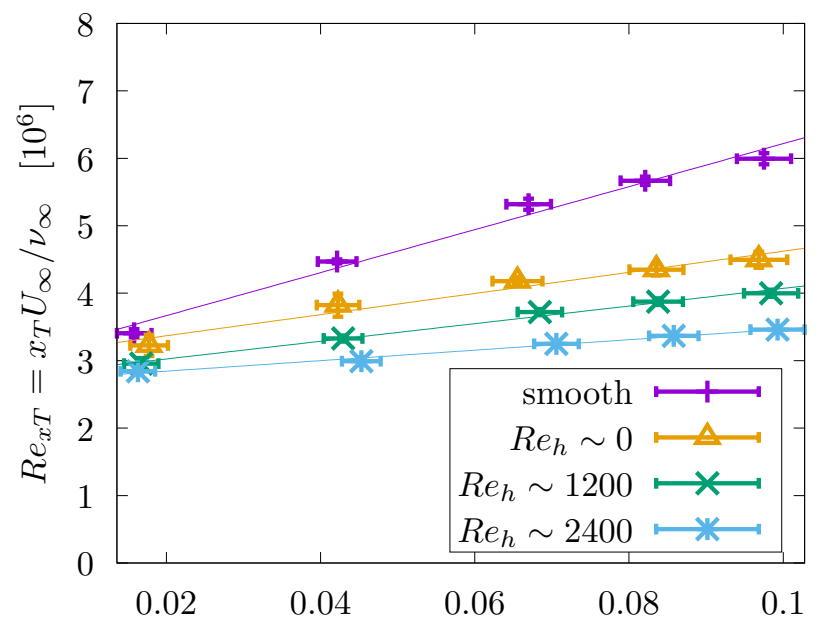

Hartree parameter $\beta_{H}$

(a) Without suction (port).

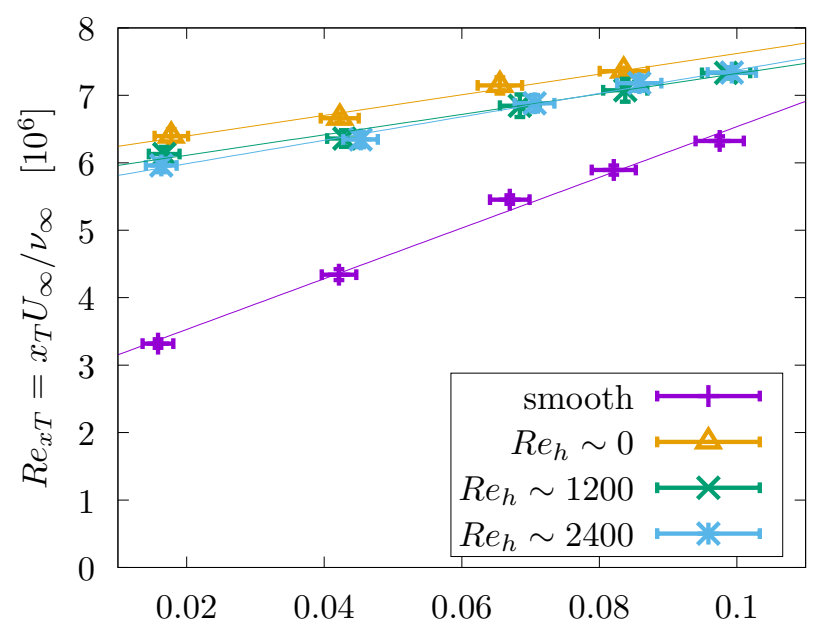

Hartree parameter $\beta_{H}$

(b) With suction (starboard).

Figure 12: Transition Reynolds number $R e_{x T}$ as function of the Hartree parameter $\beta_{H}$ for chord Reynolds number $R e_{c}=8 \cdot 10^{6}$ and Mach number $M=0.6$. Suction rate $q$ and displacement thickness $\delta^{*}$ for this data is presented in Table 2

step-induced transition location remained essentially un-550 changed for $q \gtrsim 0.2$. The suction rate $q$ can be interpreted as reduction of displacement thickness, i.e. a suction rate of $q=0.1$ results in a reduction of displacement thickness by $10 \%$ (compare ref. [17]). Table 2 presents the suction rate $q$ and displacement thickness $\delta^{*}$ for the data displayed ${ }_{555}$ in figure 12. The interpretation of $q$ as reduction of displacement thickness provides a possible explanation why

\begin{tabular}{|c|c|c||c|c|c|}
\hline \multicolumn{3}{|c||}{ step-0 $(0 \mu \mathrm{m})$} & \multicolumn{3}{c|}{ step-2 $(60 \mu \mathrm{m})$} \\
\hline$\beta_{H}$ & $q$ & $\delta^{*}[\mu \mathrm{m}]$ & $\beta_{H}$ & $q$ & $\delta^{*}[\mu \mathrm{m}]$ \\
\hline 0.018 & 0.87 & 79 & 0.016 & 1.05 & 79 \\
\hline 0.042 & 0.96 & 76 & 0.045 & 1.13 & 76 \\
\hline 0.066 & 1.05 & 73 & 0.071 & 1.22 & 73 \\
\hline 0.084 & 1.11 & 72 & 0.086 & 1.26 & 72 \\
\hline 0.097 & 1.16 & 71 & 0.099 & 1.31 & 71 \\
\hline
\end{tabular}

Table 2: Comparison of determined suction parameter $q$ and displacement thickness $\delta^{*}$ for different Hartree paramters $\beta_{H}$ for step0 and step-2 configuration. The displacement thickness $\delta^{*}$ is that at the junction location for the smooth configuration, calculated with the boundary-layer solver COCO. Chord Reynolds number is $R e_{c}=8 \cdot 10^{6}$ and Mach number is $M=0.6$. The transition Reynolds number $R e_{x T}$ for this data is displayed in figure 12

\subsection{Temperature distribution with and without suction}

The high suction rate obtained in these measurements clearly also influences the surface temperature distribution because of the different wall shear stress and therefore wall heat flux. In figure 11 the area of TSP image immediately downstream of the step on the starboard side (with suction) is slightly darker (lower temperature) compared to areas further downstream or upstream of the step and gap location. Figure 13 illustrates this temperature distribution measured with TSP, normalized with the charge temperature of the corresponding run, i.e. approximately the initial model surface temperature. The temperature 
information is averaged spanwise in the regions that were used to determine the transition location. At around 35\% chord length, the step and gap position, the model was not coated with TSP (see sec. 2.2 and therefore no temperature information could be obtained. Downstream of this area, the temperature is significantly lower for the step-0 and step-2 (suction - starboard (sb)) configurations compared to the smooth (no suction) or step-2 (no suction port $(p))$ configurations. The lower temperature can be explained with a reduced boundary-layer thickness (leading to a larger local skin friction and thus wall heat flux) and the working principle of DNW-KRG where the free stream temperature is considerately lower than the model surface temperature. This is further confirmation that the applied suction significantly decreases the boundary-layer thickness. No significant difference could be found between the step-0 (sb) and step-2 (sb) configuration even though a different suction rate $q$ was determined with $q=1.01$ for step- 0 and $q=1.17$ for step- 2 .

\subsection{Critical $N$-factors with and without suction}

In figure $14 \mathrm{a}$ the critical $\mathrm{N}$-factors for the reference configuration are displayed. In general, higher Hartree parameters $\beta_{H}$ lead to decreased critical N-factors. For low Mach numbers a higher critical N-factor was calculated $_{600}$ meaning higher amplification ratios of TS-waves are required to trigger transition [25]. This corresponds to the phenomena described in section 4.2 as of why the expected stabilizing effect of compressibility is not observed in this investigation: initial amplitudes of TS-waves are decreased for lower Mach numbers 22].

Figure $14 \mathrm{~b}$ shows the determined critical N-factors for the step-2 configuration (see section 3.2. The same trends as in figure $14 \mathrm{a}$ for the smooth configuration can be found. Interestingly, the absolute values are very similar for both cases despite the calculations in figure $14 \mathrm{~b}$ not consider-610 ing the boundary-layer prior to step and gap. In figure 15 the difference of corresponding data points (with the same

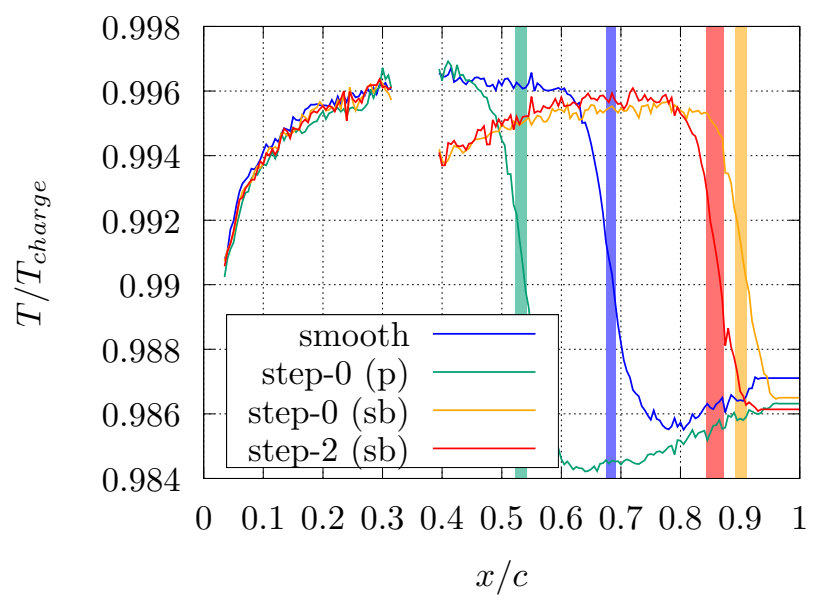

Figure 13: Chordwise temperature distribution for the smooth, step0 and step- 2 configuration on the starboard side (suction) and step-0 configuration on the port side (no suction). Colored bars mark the respective detected transition location with the width indicating the determined RMS (smooth: $x_{T} / c=0.68$, step-0 $(\mathrm{p}): x_{T} / c=0.53$, step-0 (sb): $x_{T} / c=0.90$ and step-2: $\left.x_{T} / c=0.86\right)$. Temperatures are normalized with the charge temperature of the wind tunnel, i.e. the initial model surface temperature immediately before a run. Here, chord Reynolds number is $R e_{c}=8 \cdot 10^{6}$ and Mach number is $M=0.6$ at $\beta_{H}=0.069 \pm 0.005$ for all cases.

flow conditions) in figure $14 \mathrm{a}$ and figure $14 \mathrm{~b}$ are displayed. Almost all data points have an absolute difference of critical N-factor below 1. One possible explanation is that all TS-waves amplified before the step and gap position are damped to a large extent at the step and gap location due to suction and completely new or re-amplified disturbances arise in the boundary-layer.

\subsection{Consideration on suction rates}

For flow conditions as those present in figures 11 and $12\left(R e_{c}=8 \cdot 10^{6}\right.$ and $\left.M=0.6\right)$ a suction rate of $q \approx 1$ corresponds to an average suction velocity of $v_{s u c} \approx 77$ $\mathrm{m} / \mathrm{s}$ with $v_{\text {suc }} / U_{\infty} \approx 0.38$, where $U_{\infty}$ is the freestream velocity. This is a considerable average suction velocity, but only applied in a very limited region. When considering the same massflow but for a distributed suction on the first $35 \%$ of the chord length (i.e. up to the step and gap position), this translates to $v_{\text {suc,cont. }} / U_{\infty} \approx 1 \cdot 10^{-3}$ which is typical for HLFC applications [3, 4, 39]. Distributed 


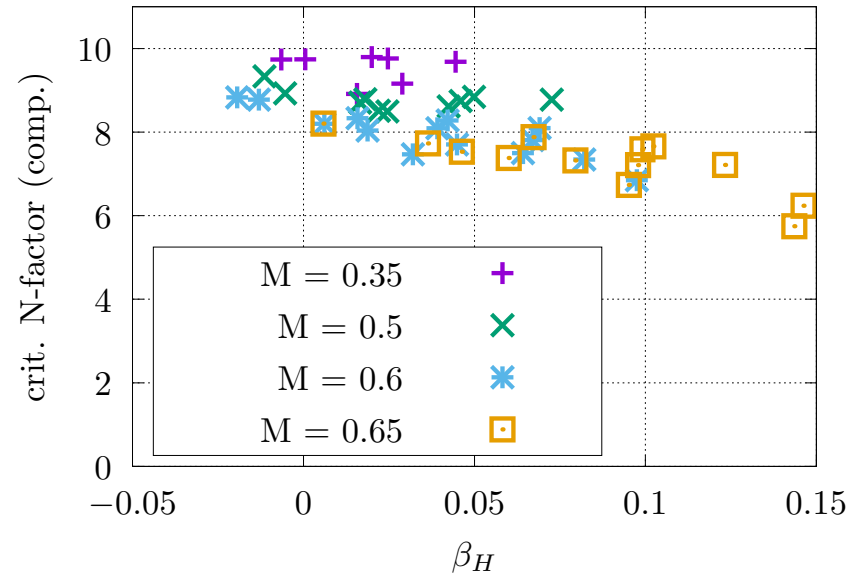

(a) smooth configuration

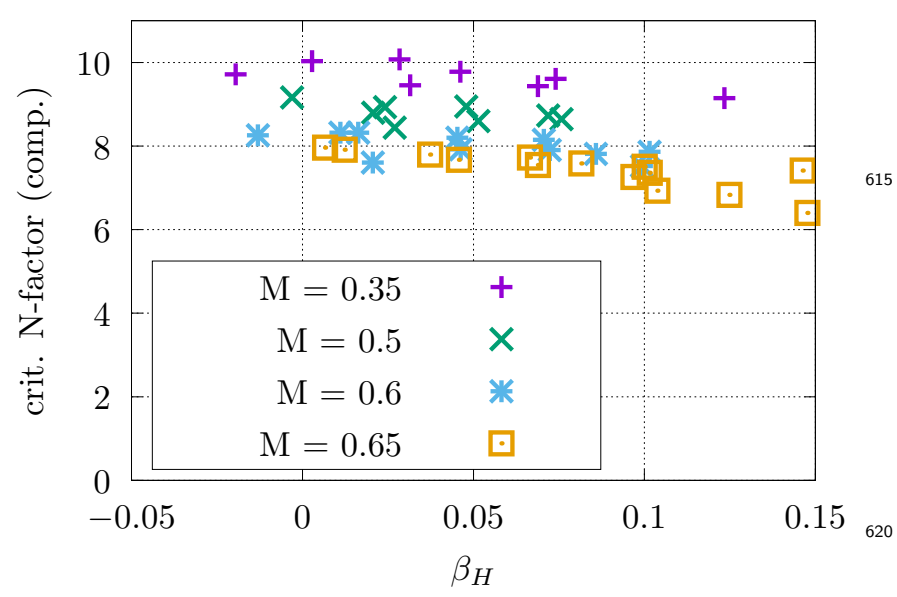

(b) step-2 configuration (approximations)

Figure 14: Computed critical N-factors (compressible) for various pressure gradient parameters at different Mach numbers (Reynolds numbers range from $3.5-12 \cdot 10^{6}$ here). (a) for the nominally smooth ${ }_{625}$ (reference) configuration and (b) for the step-2 configuration where amplification is assumed to start at the step and gap position. Absolute difference between corresponding values (with the same flow conditions) are presented in figure 15

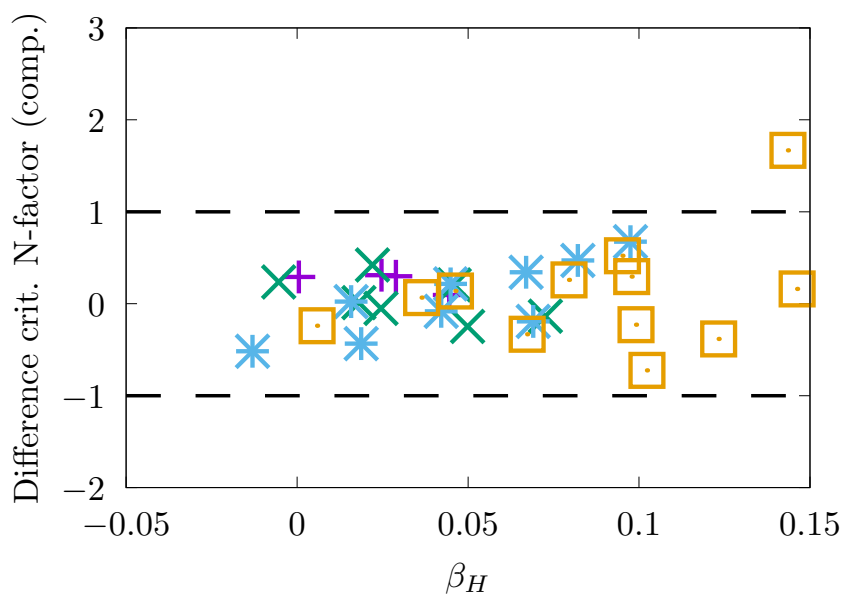

Figure 15: Difference of critical N-factors of corresponding data points in figure 14 Pressure gradient parameter $\beta_{H}$ refers to the smooth (reference) configuration. Key is the same as in figure 14 Positive values correspond to larger critical N-factors of the step-2 configuration compared to the smooth configuration.

suction hereby means suction as it is often achieved with perforated panels.

\section{Conclusions}

Experimental investigations were conducted in the Cryogenic Ludwieg-Tube Goettingen to analyze the effect of suction on step-induced boundary-layer transition. Transition was detected by means of temperature-sensitive paint on a flat-plate model in a two-dimensional flow. Mach numbers ranging from $M=0.35$ to 0.77 and chord Reynolds numbers from $R e_{c}=3.5 \cdot 10^{6}$ to $16 \cdot 10^{6}$ along with various streamwise pressure gradients were examined for three different step heights $(0 \mu \mathrm{m}, 30 \mu \mathrm{m}$ and $60 \mu \mathrm{m})$ downstream of a gap (width: $200 \mu \mathrm{m}$ ) with and without suction. Suction was achieved passively by a pressure difference between upper and lower side driving an internal flow. Pressure taps within the gap of the model were used to determine the suction rate $q$. The combination of step and gap causes transition to occur further upstream, whereas suction in the range $q \sim 1$ through the gap was found to have a significant transition-delaying effect. This was to even overcompensate the adverse effect 
of the step and gap for the examined conditions. In the

\section{Acknowlegments}

The authors would like to thank M.Rein (DLR) for ${ }^{700}$ the valuable advice during the discussion of the results, J.Bosbach (DLR) for advice in suction rate determination, A.Kunis (DLR) for the support during model modification, U.Henne (DLR) for the support of the TSP data analysis and V.Ondrus (University of Hohenheim) for the TSP development and synthesis. Furthermore, R.Kahle, M.Aschoff, A.Grimme and S.Hucke (DNW) are acknowledged for support during the measurement campaign at

\section{References}

[1] J. Robert, Drag reduction: an industrial challenge, AGARDR-786 (1992) $2-1$ to $2-15$.

[2] R. C. Wagner, D. Bartlett, F. S. Collier Jr., Laminar flow - the past, present, and prospects, AIAA Paper (1989) 1989-0989. doi https://doi.org/10.2514/6.1989-989

[3] G. Schrauf, Status and perspectives of laminar flow, The Aeronautical Journal 109 (2005) 639-644. doi https://doi.org/10. 1017/S000192400000097X

[4] S. Schaber, Auswirkungen von Schall und Absaugeverteilungen auf die Einsatzfähigkeit der Hybridlaminartechnik, Ph.D. thesis, TU Berlin, 2000.

[5] M. Fujino, Y. Yoshizaki, Y. Kawamura, Natural-laminar-flow airfoil development for a lightweight business jet, Journal of Aircraft 40 (2003) 609-615. doi https://doi.org/10.2514/1. 168

[6] A. L. Braslow, A history of suction-type laminar-flow control with emphasis on flight research, Monographs in Aerospace History, No. 13, 1999.

[7] J. D. Crouch, Boundary-layer transition prediction for laminar flow control, AIAA Paper (2015) 2015-2472. doi https://doi. org/10.2514/6.2015-2472

[8] R. D. Joslin, Aircraft laminar flow control, Annual review of fluid mechanics 30 (1998) 1-29. doi https://doi.org/10.1146/ annurev.fluid.30.1.1

[9] R. E. Kosin, Laminar flow control by suction as applied to the X-21a airplane, Journal of Aircraft 2 (1965) 384-390.

[10] D. M. Bushnell, M. H. Tuttle, Survey and bibliography on attainment of laminar flow control in air using pressure gradient and suction, NASA RP 1035 (1979).

[11] J. P. Nenni, G. L. Gluyas, Aerodynamic design and analysis of an LFC surface, Astronautics \& Aeronautics 4 (1966).

[12] M. Costantini, Experimental Analysis of Geometric, Pressure Gradient and Surface Temperature Effects on BoundaryLayer Transition in Compressible High Reynolds Number Flows, Ph.D. thesis, RWTH Aachen, 2016. URL: http://elib.dlr.de/ $117965 /$

[13] J. Perraud, D. Arnal, A. Seraudie, D. Tran, Laminar-turbulent transition on aerodynamic surfaces with imperfections, RTOAVT-111 (2004) 14-1 to 14-14.

[14] C. A. Edelmann, Influence of forward-facing steps on laminarturbulent transition, Ph.D. thesis, University of Stuttgart, 2014.

[15] C. A. Edelmann, U. Rist, Impact of forward-facing steps on laminar-turbulent transition in transonic flows, AIAA Journal 53 (2015) 2504-2511.

[16] M. Hahn, W. Pfenninger, Prevention of transition over a backward step by suction, Journal of Aircraft 10 (1973) 618-622. 
[17] J. Zahn, U. Rist, Active and natural suction at forwardfacing steps for delaying laminar-turbulent transition, AIAA Journal 55 (2017) 1343-1354. doi https://doi.org/10.2514/1. J055122

[18] J. Zahn, Control of the Impact of Steps and Gaps on Laminar-765 Turbulent Transition in Boundary Layers, Ph.D. thesis, University of Stuttgart, 2017.

[19] J. Zahn, U. Rist, Study about boundary-layer suction at a juncture for sustained laminar flow, in: A. Dillmann, G. Heller, E. Krmer, C. Wagner, S. Bansmer, R. Radespiel, R. Semaan770 (Eds.), New Results in Numerical and Experimental Fluid Mechanics XI, Springer, 2018, pp. 349-358.

[20] B. Dimond, M. Costantini, S. Risius, C. Klein, M. Rein, Experimental investigation of the delay of step-induced transition by means of suction, 21st STAB/DGLR Symposium Darmstadt,775 Germany (2018).

[21] B. Dimond, Experimental investigation of the delay of stepinduced transition by means of suction, Master's thesis, GeorgAugust-Universität, Göttingen, 2018.

[22] S. Koch, Zeitliche und räumliche Turbulenzentwicklung in780 einem Rohrwindkanal und deren Einfluss auf die Transition an Profilmodellen., Ph.D. thesis, Georg-August-Universität, Göttingen, 2004.

[23] H. Rosemann, The Cryogenic Ludwieg-Tube Tunnel at Göttingen, AGARD-R-812 (1997) 8-1 to 8-13.

[24] M. Costantini, S. Hein, U. Henne, C. Klein, S. Koch, L. Schojda, V. Ondrus, W. Schröder, Pressure gradient and nonadiabatic

\section{//doi.org/10.1007/s00348-005-1010-8}

[30] M. Costantini, S. Risius, C. Klein, Experimental investigation of the effect of forward-facing steps on boundary layer transition, Procedia IUTAM 14 (2015) 152-162. doi https://doi.org/10. $1016 / j \cdot$ piutam.2015.03.036

[31] B. Dimond, M. Costantini, S. Risius, C. Fuchs, C. Klein, Influence of suction on step-induced boundary-layer transition, in: C. J. Kähler, R. Hain, S. Scharnowski, T. Fuchs (Eds.), Proceedings of the 5th International Conference on Experimental Fluid Mechanics, 2018, pp. 167-172. doi/https://doi.org/10. $18726 / 2018 \_2$

[32] H. Dubbel, DUBBEL: Taschenbuch für den Maschinenbau, 21 ed., Springer-Verlag, 2005. doi https://doi.org/10.1007/ 3-540-26620-8

[33] G. Schrauf, COCO - a program to compute velocity and temperature profiles for local and nonlocal stability analysis of compressible, conical boundary layers with suction, ZARM Technik report (1998).

[34] G. Schrauf, LILO 2.1 users guide and tutorial, Bremen, Germany, GSSC Technical Report 6 (2006).

[35] F. Meyer, L. Kleiser, Numerical investigation of transition in 3d boundary layers, Fluid Dynamics of Three-Dimensional Turbulent Shear Flows and Transition (1989) 16-1 to 16-17.

[36] J. Perraud, A. Seraudie, Effects of steps and gaps on 2d and 3d transition, in: European Congress on Computational Methods in Applied Science and Engineering (ECCOMAS), 2000.

[37] S. Beguet, J. Perraud, M. Forte, J.-P. Brazier, Modeling of transverse gaps effects on boundary-layer transition, Journal of Aicraft 54 (2016) 794-801. doi https://doi.org/10.2514/1. C033647

[38] D. Arnal, O. Vermeersch, Compressibility effects on laminarturbulent boundary layer transition, International Journal of Engineering Systems Modelling and Simulation 3 (2011) 26-35. doi https://doi.org/10.1504/IJESMS.2011.038747

[39] H. Schlichting, K. Gersten, Boundary-layer theory, Chap 15: Onset of Turbulence (Stability Theory), 8th edition ed., Springer, Berlin, 2000. 